\title{
A Multiresonant Single-Element Wideband Slot Antenna
}

\author{
Nader Behdad, Student Member, IEEE and Kamal Sarabandi, Fellow, IEEE
}

\begin{abstract}
A new technique for designing wideband slot antennas is proposed. In this technique, the aperture's electric field distribution is manipulated to create two fictitious short circuits along the slot, hence creating two additional resonances besides the main one. The frequencies of these fictitious resonances can be chosen such that the overall bandwidth of the antenna is drastically increased. By using this technique, a slot antenna with a $1.8: 1$ bandwidth ratio is designed and fabricated. The measured results of this antenna show similar radiation patterns at different frequencies in its band of operation. Furthermore, the antenna has a relatively constant gain and more importantly, it has an excellent polarization purity over the entire bandwidth.
\end{abstract}

Index Terms-Broadband antennas, printed antennas, slot antennas.

\section{INTRODUCTION}

C URRENT advancements in printed antenna technology have resulted in a variety of different techniques for designing low profile, cost effective, and highly efficient wideband antennas. Most of these techniques, deal with marginal bandwidth improvement of more traditional antennas such as patch or printed wire antennas [1]. Another class of antennas that are suitable for miniaturization and have great reconfigurability potentials without compromising efficiency are slot antennas. However, not much attention has been paid to improving the bandwidth of this class of printed antennas. In this letter, we present a new technique for increasing the number of resonances and, hence, the bandwidth of a slot antenna by creating multiple fictitious short circuits along the slot.

Microstrip-fed wide slot antennas have been theoretically studied in [2]. Also, experimental investigations on very wide slot antennas are reported by various authors [3]-[5]. The drawback of these antennas are twofold: 1) they require a large area for the slot and a much larger area for the conductor ground plane around the slot and 2) they usually generate high cross-polarization levels that change with frequency [3]-[5]. This is mainly because these antennas can support two orthogonal modes with close resonant frequencies. The undesirable excitation of the orthogonal mode can easily occur and result in the generation of strong cross-polarized radiation. Therefore, it is important to limit the maximum width of the slot antennas

Manuscript received December 8, 2003; revised December 10, 2003. This work was supported in part by the Engineering Research Centers Program of the National Science Foundation under NSF Award EEC-9986866 and by the U.S. Army Research Office under Contract DAA-99-1-01971.

The authors are with the Radiation Laboratory, Department of Electrical Engineering and Computer Science, University of Michigan, Ann Arbor, MI 48109-2122 USA (e-mail: behdad@engin.umich.edu).

Digital Object Identifier 10.1109/LAWP.2004.825093 under investigation. It has recently been shown that a relatively wide slot antenna, fed with a narrow microstrip line, can be designed to show dual-resonance behavior with similar electric field (magnetic current) distributions at both resonant frequencies [6]. This similarity is critical since it results in similar radiation patterns and preservation of the desired polarization at different frequencies over the entire bandwidth of the antenna. The frequencies of the two resonances can be controlled in the design process such that the antenna acts as either a dual band or a broadband radiator [6]. In this letter, we will use the same concept and modify the microstrip feed to create two fictitious short circuits along the slot with close enough frequencies to obtain a much wider bandwidth. By using this technique, a single-element slot antenna with a bandwidth as high as $1.8: 1$ is designed and fabricated. This antenna shows similar radiation patterns with very low levels of cross-polarized radiation over the entire bandwidth. In what follows, first the design procedure is studied and then the measured results are presented and discussed.

\section{Design PROCEDURE}

A relatively wide slot antenna, fed with a narrow microstrip line near an edge, can be designed to show a dual-resonance behavior, which can be exploited to obtain a wideband response [6]. When a relatively wide slot antenna is fed with a narrow microstrip line, the electric field generated by that part of the microstrip line over the slot (which does not have a ground plane), cancels the slot electric field, generated by the return current of the microstrip-line in the ground plane, at a certain location near the feed. This creates a fictitious short circuit along the slot near the microstrip feed and, hence, generates a fictitious resonance with a frequency, which is slightly higher than that of the main resonance [6]. The next challenging step is to examine whether it is possible to create more than one fictitious short using a similar approach or not. If this is possible, the bandwidth of the antenna can drastically be increased by merging these fictitious resonances. In order to test the validity of this idea, we begin by attempting to place two fictitious short circuits along the slot, using a two-prong microstrip feed. It is expected that this structure could present three distinct resonances at frequencies proportional to the resonant lengths $L_{r 1}, L_{r 2}$, and $L_{r 3}$ as shown in Fig. 1. If the locations of the two feed lines are chosen properly, the three resonant frequencies will be close and the overall bandwidth of the antenna can be increased significantly by using an appropriate feeding network. The location of the microstrip feeds, $L_{s 1}$ and $L_{s 2}$ in Fig. 2, determines $L_{r 2}$ and $L_{r 3}$ and, hence, the resonant frequencies of the two fictitious resonances. 


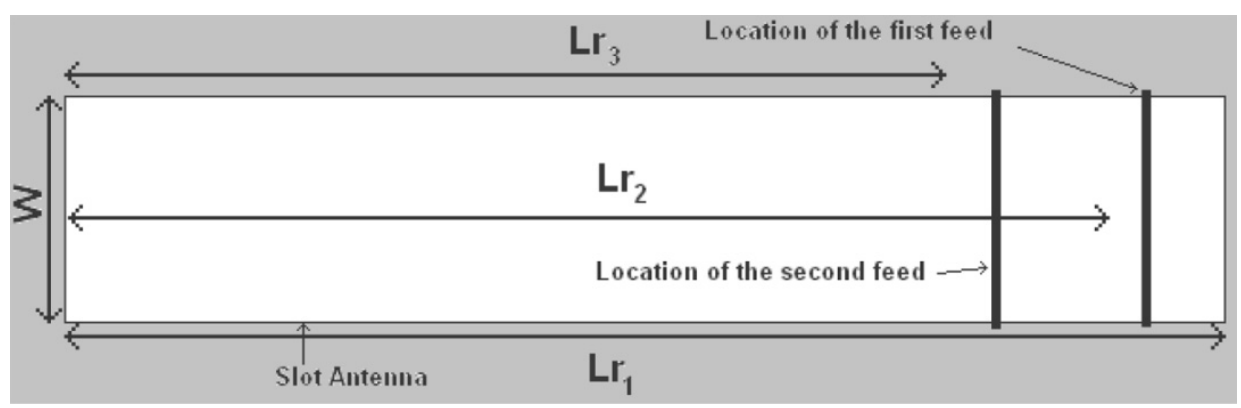

Fig. 1. Schematic of the relatively wide slot antenna and its equivalent resonant lengths.

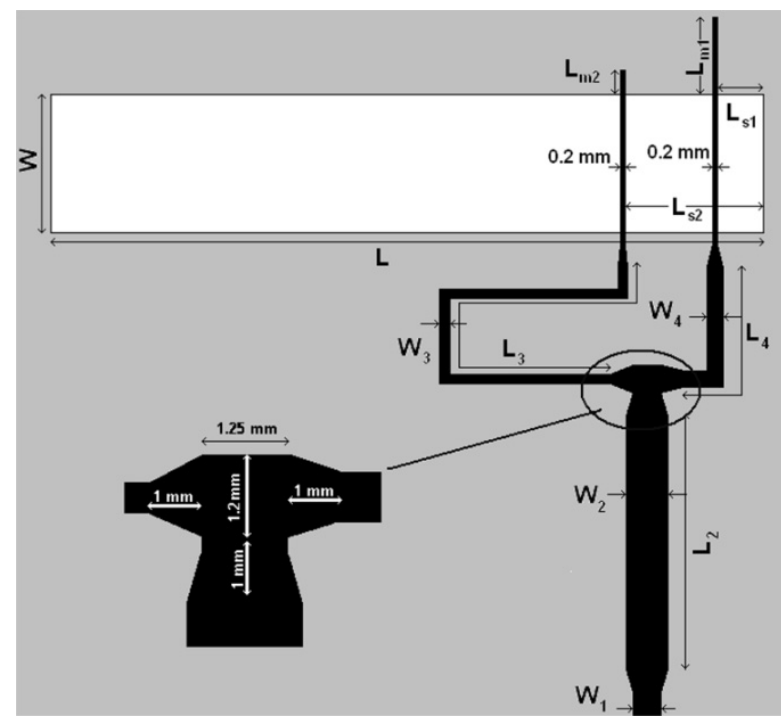

Fig. 2. Schematic of the broadband slot antenna and its microstrip feed network.

The feed topology chosen for this antenna (Fig. 2) can be viewed as a three-port microstrip network that achieves power division using a microstrip-Tee. In order to obtain a broadband response, matching is performed by choosing the feed network parameters, $L_{2}, W_{2}, L_{3}, W_{3}, L_{4}, W_{4}, L_{s 1}, L_{s 2}, L_{m 1}$, and $L_{m 2}$ (shown in Fig. 2) appropriately. These parameters must be chosen such that the overall impedance bandwidth of the antenna is maximized. In order to obtain the optimum values of these parameters, a combined full-wave and network simulation technique is used. First, the antenna structure and its narrow microstrip feed lines (Fig. 1) are simulated as a four port network using a full-wave electromagnetic (EM) simulation tool [8]. Then the S-parameters of this four-port network are used in a network simulation and optimization software [9] from which the optimized values of the feed-network parameters are obtained. This technique allows for rapid optimization of the network parameters, but it ignores the coupling effects that exist between different components of the feed network. Therefore, a final full-wave simulation of the structure is performed and the different feed parameters are fine tuned to obtain a good response. The final schematic of the antenna is shown in Fig. 2, and the optimized dimensions of the antenna are given in Table I.
TABLE I

A SuMmary of THE PHySiCAL Dimensions of THE BROADBAND Single-Element Slot AntenNa. All Dimensions are in Millimeters

\begin{tabular}{c||c|c|c|c|c|c}
\hline Parameter & $L$ & $W$ & $L_{2}$ & $W_{2}$ & $L_{3}$ & $W_{3}$ \\
\hline Value & 31 & 6 & 11 & 1.8 & 19.73 & 0.43 \\
\hline Parameter & $L_{4}$ & $W_{4}$ & $L_{s 1}$ & $L_{s 2}$ & $L_{m 1}$ & $L_{m 2}$ \\
\hline Value & 6.2 & 0.72 & 2 & 6 & 3.3 & 1
\end{tabular}

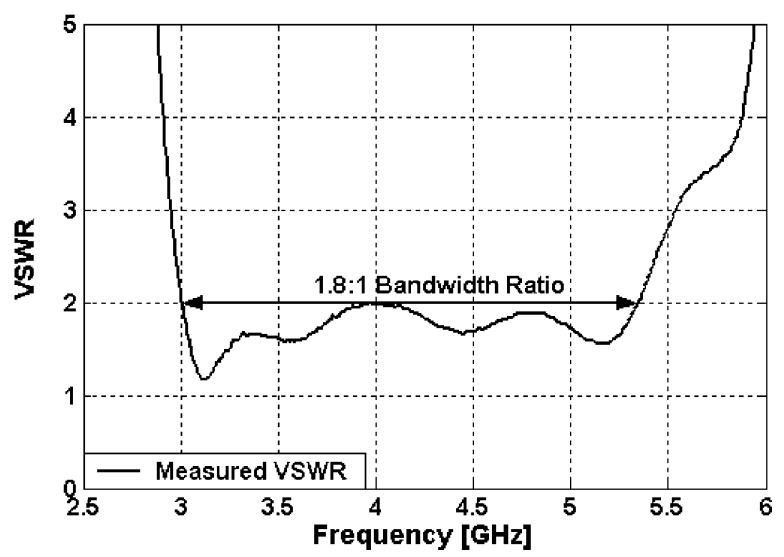

Fig. 3. Measured VSWR of the broadband slot antenna.

\section{MEASUREMENT RESUlTS AND DISCUSSION}

The proposed antenna is fabricated on a 500- $\mu \mathrm{m}$-thick RO4350B substrate with a dielectric constant of $\epsilon_{r}=3.4$ and $\tan \delta$ of 0.003 with a ground plane size of $11.5 \mathrm{~cm} \times 10 \mathrm{~cm}$. The voltage standing wave ratio (VSWR) of the antenna is measured using a calibrated vector network analyzer and is shown in Fig. 3. It is shown that the VSWR of the antenna remains below 2 over a bandwidth range of 3 to $5.4 \mathrm{GHz}$. This corresponds to a 1.8:1 impedance bandwidth ratio. However, in order to determine the overall bandwidth of the antenna, other radiation parameters such as radiation patterns, gain, and antenna polarization must also be carefully examined over the entire frequency band. The radiation patterns of the antenna are measured at five different frequencies in the anechoic chamber of the University of Michigan. For brevity, the patterns at only three frequencies are given in Fig. 4. It is observed that the radiation patterns at different frequencies are similar, which is expected from a wideband antenna. More importantly, the cross-polarization levels are very small for all the measured 


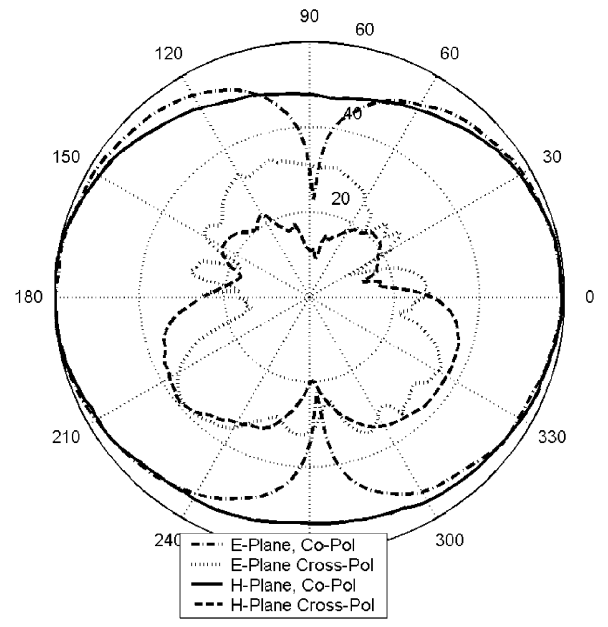

(a)

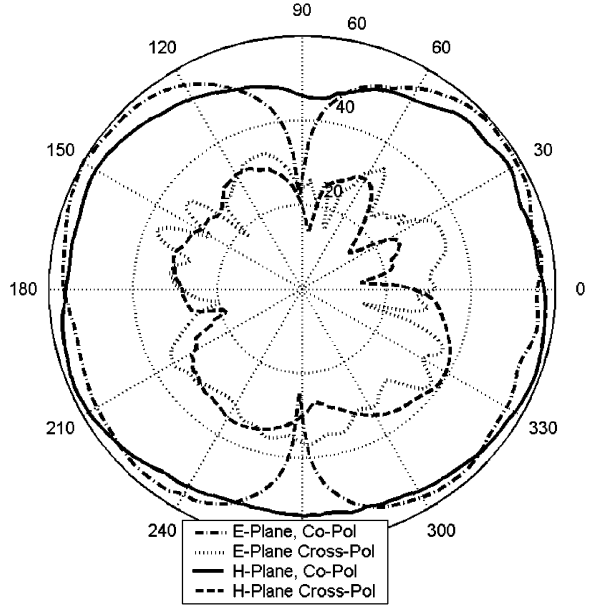

(b)

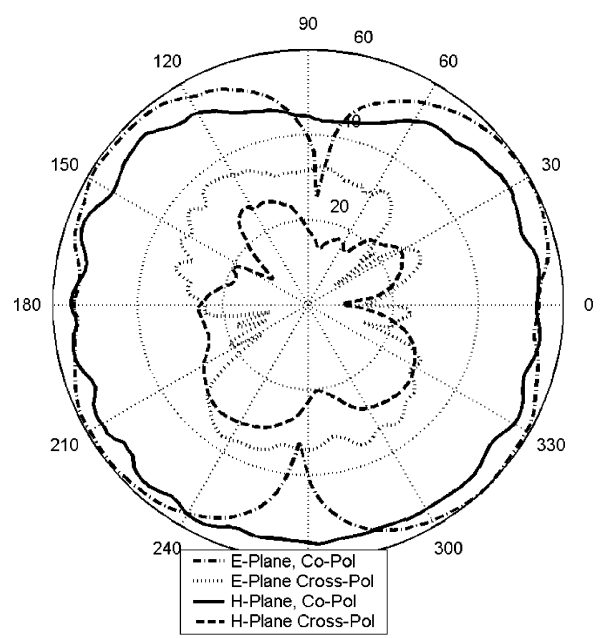

(c)

Fig. 4. Radiation patterns of the broadband slot antenna, measured at (a) $f=3 \mathrm{GHz}$, (b) $f=4 \mathrm{GHz}$, and (c) $f=5 \mathrm{GHz}$.

patterns. Specifically, a Co-Pol/Cross-Pol ratio of better than $30 \mathrm{~dB}$ is observed at boresight in all of the measured radiation patterns. The cross-polarization levels at other directions are also very small, indicating excellent polarization purity.

As can be observed from Fig. 4, the patterns of the antenna are not the dual of those of an electric dipole. The first difference is that the patterns in the $E$-plane (unlike the $H$-plane of an electric dipole) show two nulls at $\pm 90^{\circ}$, which are caused by two different mechanisms. The first mechanism is the cancellation that occurs at grazing angle as a result of the phase difference between $E$-fields on the top and bottom of the ground pland. This phase difference does not exist in the $H$-plane. Furthermore, the $H$-plane radiation pattern should inherently show two nulls at $\pm 90^{\circ}$ as a result of the boundary condition that forces the tangential component of the electric field to go to zero. The second reason for having these nulls is that the slot antenna is covered with a dielectric substrate at one side. This forces the normal component of the electric field at grazing angles to go to zero as described in [7]. Another difference is the minima (instead of nulls) in the $H$-plane which is a direct result of the radiation from the edges of the ground plane. The antenna gain is measured in the anechoic chamber using a standard double ridge horn and is presented in Fig. 5. It is seen that the gain of the antenna remains relatively constant over the entire bandwidth. Based on the measured VSWR, radiation patterns, polarization purity, and gain of the antenna, the overall bandwidth is determined to be the same as its impedance bandwidth (1.8:1). Preserving the radiation patterns and excellent polarization purity are extremely important factors in designing wideband antennas. Since many of the existing wideband, single-element, printed antennas achieve wideband operation from different radiation mechanisms and field distributions at different frequencies, they cannot easily achieve these two important factors. This letter presents a very simple way of designing a wideband printed antenna with excellent radiation parameters over the entire bandwidth.

\section{CONCLUSION}

A new and simple technique for designing wideband slot antennas is presented. This technique is based on creating a number of fictitious resonances along the slot with close frequencies and using them to obtain a wideband overall response with similar radiation patterns and polarization over the entire bandwidth of the antenna. This is accomplished 


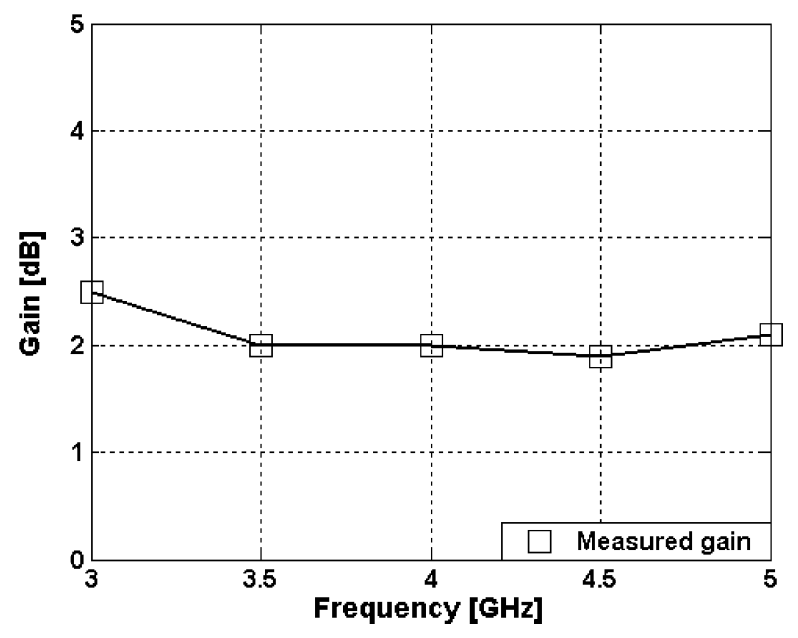

Fig. 5. Measured gain of the broadband slot antenna. Note that maximum measured gain in the azimuthal plane is reported.

using a two-prong microstrip feed on a single slot antenna and is shown to provide a $1.8: 1$ bandwidth as well as very low cross-polarization levels at different directions and frequencies.
Simplicity of the design process, consistent radiation parameters, and excellent polarization purity make this technique very attractive for designing wideband printed antennas.

\section{REFERENCES}

[1] G. Kumar and K. P. Ray, Broadband Microstrip Antennas. Norwood, MA: Artech House, 2002.

[2] M. Kahrizi, T. Sarkar, and Z. Maricevic, "Analysis of a wide radiating slot in the ground plane of a microstrip line," IEEE Trans. Microwave Theory Tech., vol. 41, pp. 29-37, Jan. 1993.

[3] J. Sze and K. Wong, "Bandwidth enhancement of a microstrip-line-fed printed wide slot antenna," IEEE Trans. Antennas Propagat., vol. 49, pp. 1020-1024, July 2001.

[4] H. L. Lee, H. J. Lee, J. G. Yook, and H. K. Park, "Broadband planar antenna having round corner rectangular wide slot," in Proc. IEEE Antennas and Propagation Society Int. Symp., vol. 2, San Antonio, TX, June 2002, pp. 460-463.

[5] P. H. Rao, V. Fusco, and R. Cahill, "A broadband antenna for PCN/UMTS applications," High Freq. Postgrad. Student Colloq., pp. 2-5, Sept. 2000.

[6] N. Behdad and K. Sarabandi, "A novel approach for bandwidth enhancement of slot antennas," in Proc. Antenna Applications Symp., Monticello, IL, Sept. 17-19, 2003, pp. 176-189.

[7] C. A. Balanis, Antenna Theory: Analysis and Design, 2nd ed. New York: Wiley, 1996, ch. 12.

[8] IE3D Electromagnetic Simulation and Optimization Software.

[9] Advanced Design System. 